\title{
Serum melatonin levels in pharyngo-laryngeal carcinomas
}

Adina Zamfir Chiru Anton"1, Dana Manda 2, Radu Cristian Popescu³, Gheorghe Dan Cristian

\author{
${ }^{1}$ Childrens' Hospital "Grigore Alexandrescu" Bucharest, \\ ${ }^{2}$ National Institute of Endocrinology "C.I.Parhon" Bucharest, \\ 3"Coltea" Clinical Hospital, Bucharest, \\ ${ }^{4}$ Childrens' Hospital M.S. Curie Bucharest, Romania
}

Melatonin is mostly known as a circadian hormone, but it also has effects in a large number of cellular processes (cell differentiation, proliferation, apoptosis and redox balance). Although it was studied in many tumours, very little is known about his role in pharyngo-laryngeal carcinomas.

Aim: to evaluate serum melatonin levels in patients with pharyngo-laryngeal malignant tumours preoperatively and after surgical excision of the malignancy.

Subjects and methods: 45 patients ( 41 men and 4 women, $59 \pm 7.42$ years) and 35 age-matched healthy subjects have been tested for melatonin levels using ELISA method after serum extraction by reverse phase chromatography. Each patient had two blood samples, one in the morning and one at midnight in order to record the day and night melatonin levels. 19 patients $(42.2 \%)$ presented a laryngeal squamous cell carcinoma, 22 patients $(48.8 \%)$ pharyngeal, base of tongue or tonsil squamous cell carcinoma, and 4 patients (9\%) had other malignant tumours in the head and neck region. Patients with $\beta$-adrenergic blockers medication have been excluded.

Results: $43.7 \%$ of the patients had a T1 stage tumour, while T2, T3 and T4 stages were recorded in $18.75 \%, 25.05 \%$ and $12.5 \%$ respectively. Biopsy revealed a $82,9 \%$ of squamous cell carcinoma in $82.9 \%$ of the patients. Tumour patients have been followed monitored postoperatively and their melatonin secretion tested after surgery as well ( 2 days after the surgery). Before surgery, cancer patients had significant lower levels of melatonin compared to noncancer healthy subjects. There were no significant changes immediately after surgical removal of the tumors.

There were no significant differences between different $\mathrm{T}$ or $\mathrm{N}$ score subgroups.
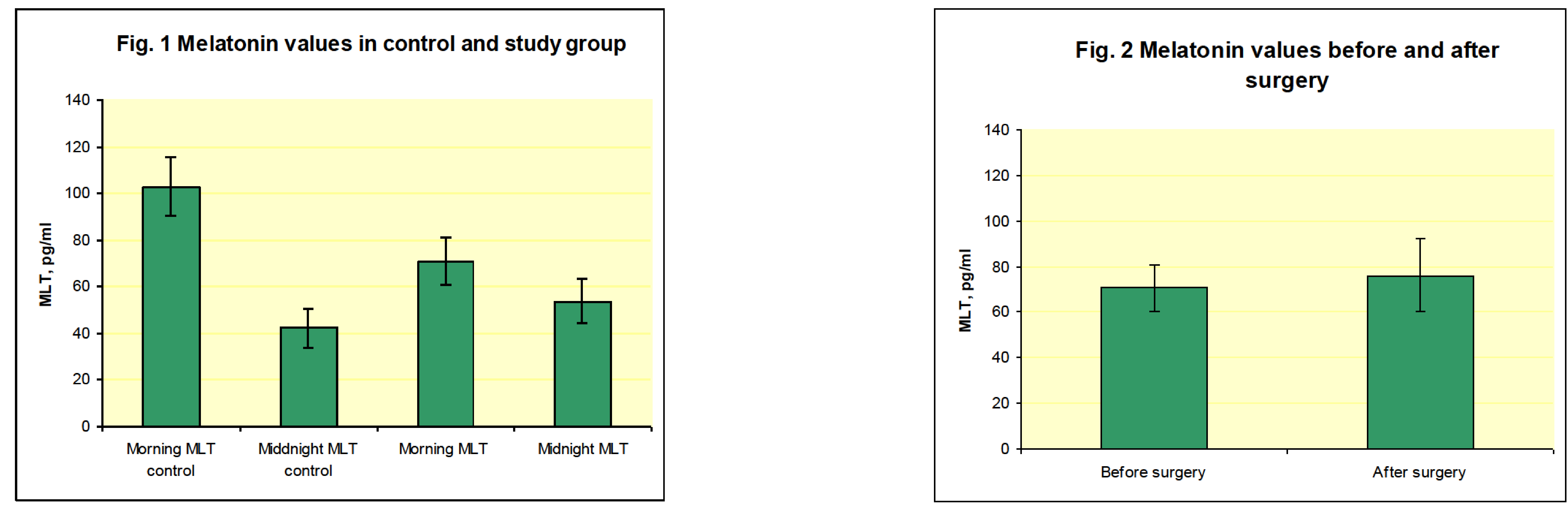

Conclusion: Serum melatonin levels are lower in patients harbouring a pharyngeal or laryngeal tumour compared to healthy subjects. The idea of long-term recording of melatonin levels in head an neck cancer patients treated by different therapeutical approaches and future possibilities of using melatonin substitution in therapy is discussed. 